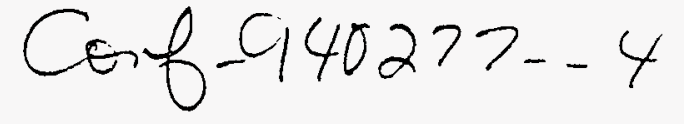

\title{
Turbulent Dispersion of Particles: The STP Model
}

\author{
L.L. Baxter*广 and P.J. Smith§ \\ Brigham Young University \\ Provo, Utah 84602
}

\author{
*Author to whom correspondence should be sent. \\ †Current Address: \\ Combustion Research Facility \\ Livermore, CA 94550 \\ $\S$ Current Address: \\ Department of Chemical and Fuels Engineering \\ University of Utah \\ Salt Lake City, UT 84112
}

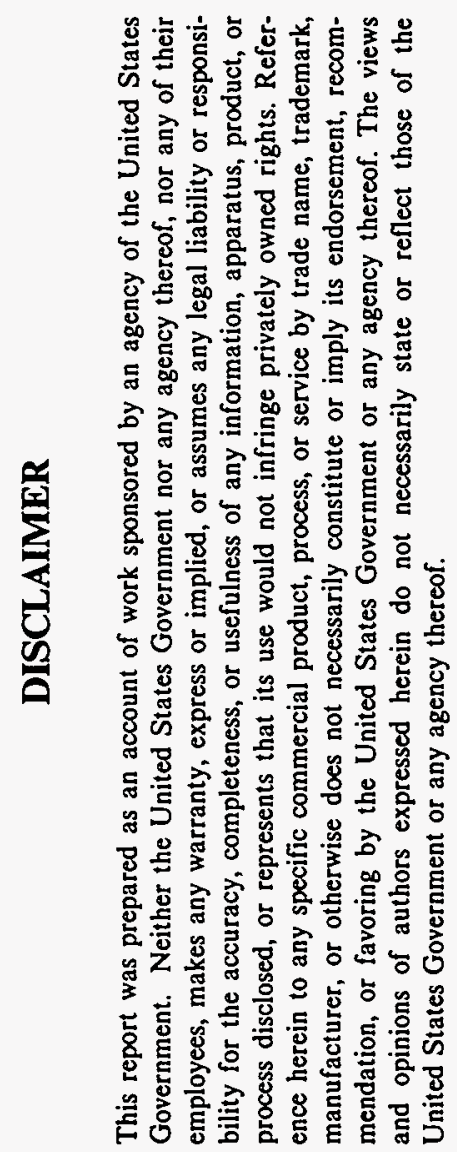

\section{Abstract}

A mathematical description of the stochastic transport of particles (STP) model for particle dispersion in turbulent flows is presented. The STP model is based on established theories of stochastic process modeling. The parameters of the model include physical properties of the particle (diameter and mass) and a description of the turbulent characteristics (mean velocities with rms fluctuations and their residence-time correlations) of the fluid phase in which the particles are dispersed. The model includes no adjustable parameters in the sense of calibration factors. It is independent of any particular turbulence model, but estimates of its parameters from information available from the common $k-\varepsilon$ turbulence model are presented.

Elements of the STP model are compared with exact solutions of the diffusion equation, alternative dispersion models, and experimental data collected under well-defined conditions. The STP model reproduces the exact solutions when both are based on consistent assumptions. Suggested 


\section{DISCLAIMER}

Portions of this document may be illegible in electronic image products. Images are produced from the best available original document. 
approximated terms in the model also reproduce the experimental data nearly within its error under simple flow conditions. The STP model describes the origin of more complex behavior (countergradient diffusion, for example) and has the potential of describing it if sufficient detail about the gas-phase turbulence is known.

\section{Introduction}

The fluid mechanics of most processes that entrain particles, droplets, or slurries as a dispersed phase in a gas stream are turbulent. Examples include spray drying, pneumatic transport of particles, heterogeneous chemical reaction/catalysis systems, and a large variety of internal and external combustion systems. The interactions of the condensed phases with the turbulent fluid mechanics in such systems are difficult to characterize. Characterization of the gas phase turbulence in itself is difficult, even in the absence of condensed phases. But the complications in describing the dispersion and reaction of the condensed phases in turbulent environments do not stem entirely or even primarily from the uncertainties in the description of the turbulence. Theoretical descriptions of the turbulent dispersion of particles and droplets are not well established, even when the characteristics of the gas-phase turbulence are known.

The focus of this paper is the description of the transport of particles in turbulent gas flows. Throughout the discussion, the properties of the gas phase turbulence will be assumed known by assumption, experimental measurement, or from a model. Accurate measurements and models of turbulence properties in complex, developing flows are not generally available and the inaccuracies of available data and models will affect the results of turbulent transport modeling. However, the intent of this paper is to separate the gas phase turbulence modeling problem from particle transport in turbulent flows and explore a method of describing the latter if the former is well characterized.

Several reviews of turbulent particle dispersion document the progress in this area of research to date. Reviews of mixing and transport in turbulent environments and various aspects of the

turbulent dispersion problem are available ${ }^{1-8}$. In terms of an analytical and comprehensive 
description of turbulent dispersion, Shuen and coworkers 9 have shown modifications of the stochastic separated flow model (SSF) ${ }^{10}$ which yields reasonably good predictions in a variety of flows and may be the most rigorous technique currently available. The SSF model is based on a Monte-Carlo simulation of the particle flow field. Several thousand trajectories are typically used to compute the overall particle or droplet behavior. The success of this approach is also dependent on a somewhat arbitrary choice of methods of evaluating its parameters. This and the host of other models suggested in the literature also depends on an accurate description of gas-phase turbulence.

The following discussion presents a description of the turbulent dispersion of particles which differs from currently employed models It is derived from theories of stochastic proceesses such as Brownian motion and classical turbulent dispersion models with established historical development ${ }^{1-15}$. The characteristics of the gas-phase turbulence are assumed to be known, either from experiment or from an appropriate model. Current gas turbulence models and experiments sometimes fall short of producing reliable estimates of turbulence properties in complex flows. The STP model uses classical properties of turbulence directly in its formulation and is independent of any particular turbulence model. The STP model will benefit from future improvements, and be limited by current inaccuracies, in the parameters used to characterize the properties of the flow.

This following discussion conceptually introduces the STP model, summarizes the mathematical equations on which it is based, compares its predictions with theory and data, and sicusses implications of the results for particle dispersion generally.

\section{Conceptual Illustration of the STP Model}

The approach to describing turbulent particle dispersion in the STP model is to predict the statistics for the location of a cloud of identical particles released from a single point into a steady but inhomogeneous flow. Conceptually, the position of an individual particles is monitored as a function of residence time. Many such particles are released in sequence, generating a large 
number of particle position histories (trajectories). The statistics of these individual trials define a probability density function (pdf) for particles position that changes with residence time. This pdf is described in terms of time-dependent statistical parameters, such as the average particle position, the average standard deviation in particle position, correlation coefficients, and similar statistics.

Figure 1 presents an illustration of such a pdf early in residence time in an arbitrary twodimensional flow field. The average particle position is near the origin, with a relatively small amount of dispersion about this mean. This represents the pdf shortly after the particles have been injected from a point source near the origin. Figure 2 illustrates the same particles later in residence time. The maximum value of the pdf is lower (note scale change on probability density axis) and the amount of dispersion is greater. The mean position is located at the $(x, y)$ point of about $(4.5,2)$. Figure 3 illustrates the pdf shortly before the mean leaves the flow field. Probability contour lines are superimposed on the bottom of this figure as a series of ovals. In this case, the extent of particle dispersion reaches completely across the flow domain in the y direction and well past the end of the flow domain in the $\mathrm{x}$ direction.

A summary of the changing pdf is presented in schematic form in Figure 4. Here, each circle represents a contour line of the pdf at a different residence time. As is illustrated, the pdf gets continuously larger. The location of a typical Eulerian computational cell is also indicated in the figure. Several of the circles overlap the particular cell. This implies that particles of several different residence contribute to the overall particle contribution of the cell. The STP model tracks the time development of the pdf and the contributions to mass, momentum, and energy that the particles make within each computational cell.

In this paper, the basis for the time development of parameters describing the pdf is summarized. The implementation of the equations in a computational fluid dynamics model is completed ${ }^{16}$, but not discussed here. The discussion here focuses on development of the model, illustration of important model predictions by comparison with other models and data, and discussion of implications of the results for modeling of turbulent particle dispersion generally. 


\section{Theoretical Discussion}

The details of the stochastic model of turbulent particle dispersion, the STP model, are discussed in detail elsewhere ${ }^{16}$. The model is presented in summary form below in a form appropriate for a large number of experimental and theoretical conditions. The assumptions required to derive these equations include (1) that the instantaneous particle and gas velocities are related through a linear differential equation (e.g., by Stokes' law), (2) that the correlation between particle number density and gas-phase velocity fluctuations is known, (3) that the mean and fluctuating components of the gas velocities are known everywhere in the flow field, and (4) that the turbulent properties change in the flow field at a rate comparable to or less than the relaxation time of the particles. One of the driving forces for this work is the description of the particle phase in relatively comprehensive pulverized coal combustion models. Assumptions 1 and 4 are well justified under these conditions (small particles with modest density dispersed in modestly turbulent, essentially steady-state gas flows). The supposition in developing the STP model is that the gas phase can be described either by experiment or, more commonly, by a computational fluid dynamics model that is to be coupled with the STP model. Assumption number 3, the coupling between particle concentrations and gasphase velocity fluctuations, addresses a level of detail rarely available from either experiment or modeling. The assumption commonly used in applications of the STP model is that there is no correlation between the two. This is an oversimplification, particularly in the early stages of particle dispersion, for many conditions. However, there appear to be no reasonable alternatives in most applications of the model. Relaxation of all of these assumptions can be done within the framework of the STP model without great difficulty if sufficient detail is known about the gasphase behavior, as is discussed elsewhere ${ }^{16}$. The discussion here focuses on application of the model within the limitations of commonly used descriptions and measurements of gas-phase turbulence, i.e., turbulence models or data that detail mean gas-phase velocities and the rms values of their fluctuations as a function of position and time.

\section{General Equations for the STP Model}


The STP model describes particle dispersion as a stochastic process, and its equations describe statistical properties of the dispersion process. In particular, the equations describe the mean and covariance matrix for particle position as a function of particle residence time, designated $t_{2}$. The time of release of particles is given as $t_{1}$. Transient processes and individual particle motion are functions of both $t_{1}$ and $t_{2}$. For steady (statistically stationary) flows, the mean and covariance matrix for particle position are dependent on particle residence time but not on the time at which particles are released. The equation for mean particle position is

$$
\left\langle x_{i}\left(t_{1}, t_{2}\right)\right\rangle \equiv \int_{0}^{t_{2}}\left\langle u_{i}\left(t_{1}, t\right)\right\rangle d t+\left\langle x_{i}\left(t_{1}, t_{2}=0\right)\right\rangle
$$

where $x$ and $v$ represent position and velocity, respectively, and angle brackets represent ensemble average values. Traditional indicial notation is used to represent the particle position vector, with subscripts $i$ and $j$ representing coordinate directions here and elsewhere. In Eq. $1, x_{j}$ represents the position vector of an individual particle and is a function of both $t_{1}$ and $t_{2}$. The ensemble average of $x_{i}$ is an average with respect to time of release $\left(t_{1}\right)$ and depends only on residence time $\left(t_{2}\right)$.

The particles whose positions are represented by $x_{i}$ in Eq. 1 can be either fluid particles or macroscopic particles. Macroscopic particle properties are represented hereafter by a subscript $p$, which is separated from the indicial subscripts $i$ and $j$ by a semicolon. The equation of motion for macroscopic particles is given by

$$
\frac{d u_{i ; p}\left(t_{1}, t_{2}\right)}{d t_{2}}=\beta\left(t_{2}\right)\left(u_{i ; g}\left(t_{1}, t_{2}\right)-u_{i ; p}\left(t_{1}, t_{2}\right)\right)+A_{i}\left(t_{1}, t_{2}\right)+g_{i}
$$

where $\beta$ represents the exchange coefficient, $A$ represents random forces normalized by particle mass such as molecular collisions (which give rise to Brownian motion), $g$ represents the acceleration of gravity (or any other body forces normalized by mass), and the other terms have been previously defined. In many practical cases, the exchange coefficient is independent of particle and gas velocity. For example, for particles that obey Stokes' law the exchange coefficient 
in given by $3 \pi d_{p} \mu_{g} / m_{p}$. The residence-time dependence of the exchange coefficient accounts for the changes in particle mass and diameter and gas viscosity (temperature and composition) for reacting particles in a reacting flow. Release-time dependence of the exchange coefficient is ignored in Eq. 2; identical particles are assumed to be released into a fluctuating velocity field, but changes in the velocity field are assumed to have no impact on the exchange coefficient.

Eq. 2 contains few assumptions for nonreacting particles in isothermal flows. Addional terms can be added to Eq. 2 to describe virtual mass, thrust, Magnus, Bassett, and Saffman forces, buoyancy, etc. ${ }^{2,17}$ The combined contributions of these terms is small (typically less than $1 \%$ ) in most practical applications ${ }^{16}$, so they are neglected in the remaining discussion here. In applications where they are significant, they could be included without change in the general approach to the problem.

The ensemble average of Eq. 2 provides a basis for calculating the mean particle position. Particle clouds with exchange coefficients that are uncorrelated with initial time, particle velocity, and gas velocity exhibit mean positions given by

$$
\frac{d\left\langle u_{i ; \rho}\left(t_{1}, t_{2}\right)\right\rangle}{d t_{2}}=\beta\left(t_{2}\right)\left(\left\langle u_{i ; g}\left(t_{1}, t_{2}\right)\right\rangle-\left\langle u_{i: \rho}\left(t_{1}, t_{2}\right)\right\rangle\right)+\left\langle A_{i}\left(t_{1}, t_{2}\right)\right\rangle+g_{i}
$$

Residence-time variations in the exchange coefficient are assumed to be independent of release-time variations in gas properties in Eq. 3. The ensemble average of the gas velocity can be calculated from its expected value, given by

$$
\left\langle u_{i ; g}\left(t_{1}, t_{2}\right)\right\rangle=\frac{\iiint \int_{x_{i}= \pm \infty}\left[\left\langle u_{i ; g}\left(x_{i}, t_{1}, t_{2}\right)\right\rangle\left\langle n\left(x_{i}, t_{1}, t_{2}\right)\right\rangle\right] d x_{i}}{n_{T}}
$$

where $n$ represents particle number density. This is not the same as the average gas velocity at the mean particle position, although the latter can be shown to be a leading term in the calculation. Ensemble average values for the components of the random force can be similarly computed. 
The difference between these means and the instantaneous values of the corresponding variables is designated by a prime and represents a perturbation from the mean. For a generic vector $v$,

$$
v_{i}\left(t_{1}, t_{2}\right)=\left\langle v_{i}\left(t_{1}, t_{2}\right)\right\rangle+v_{i}^{\prime}\left(t_{1}, t_{2}\right)
$$

This decomposition of the variables differs in concept and magnitude from the Reynolds and Favre averages of Eulerian properties discussed in most turbulence literature. Equation 5 includes an ensemble average, or expected value, of a Lagrangian variable for a cloud of particles. This ensemble average is computed by conducting an experiment with many different release times and averaging the results as a function of residence time. Such an average is mathematically identical to the expected value, which is computed by integration over a pdf, as indicated in Eq. 4 . The ensemble average is independent of release time if the Eulerian flow variables are stationary (flow is at steady state). The Reynolds- and Favre-averaged quantities in most turbulence literature relate to point measurements. These averages result from time averaging the variable (Reynolds average) or the product of the variable and the density (Favre average) at one point in space.

The solution to Eq. 3 yields the mean velocity of the particle cloud, the integral of which defines the mean position. The rate at which the cloud expands is defined by the covariance matrix. The time rate of change of the particle velocity covariance tensor is computed by ensemble averaging the product of the position perturbations. The result yields

$$
\begin{aligned}
\frac{d\left\langle x_{i ; p}^{\prime}\left(t_{1}, t_{2}\right) x_{j ; p}^{\prime}\left(t_{1}, t_{2}\right)\right\rangle}{d t_{2}}= & \int_{0}^{t_{2}}\left\{\left\langle u_{i ; p}^{\prime}\left(t_{1}, \tau\right) u_{j ; p}^{\prime}\left(t_{1}, t_{2}\right)\right\rangle+\left\langle u_{j ; p}^{\prime}\left(t_{1}, \tau\right) u_{i ; p}^{\prime}\left(t_{1}, t_{2}\right)\right\rangle\right\} d \tau+ \\
& \left\langle x_{i ; p}^{\prime}\left(t_{1}, t_{2}=0\right) u_{j ; p}^{\prime}\left(t_{1}, t_{2}\right)\right\rangle+\left\langle x_{j ; p}^{\prime}\left(t_{1}, t_{2}=0\right) u_{i ; p}^{\prime}\left(t_{1}, t_{2}\right)\right\rangle
\end{aligned}
$$

Particles released from a point source have variations in the position of particle release, $x_{i}^{\prime}\left(t_{1}, t_{2}=0\right)$, of zero. Under these common conditions, the last two terms in Eq. 6 can be dropped. 
Normalization of the velocity correlations in Eq. 6 by $\left\langle u_{i, p}^{\prime 2}\left(t_{1}, t_{2}\right)\right\rangle^{1 / 2}\left\langle u_{j: p}^{\prime 2}\left(t_{1}, t_{2}\right)\right\rangle^{1 / 2}$ and defining a normalized Lagrangian correlation tensor as

$$
R_{i j ; p}^{L}\left(\tau, t_{2}\right) \equiv \frac{\left\langle u_{i ; p}^{\prime}\left(t_{1}, \tau\right) u_{j ; p}^{\prime}\left(t_{1}, t_{2}\right)\right\rangle}{\left\langle u^{\prime 2}{ }_{i ; p}\left(t_{1}, t_{2}\right)\right\rangle^{1 / 2}\left\langle u^{\prime 2}{ }_{j ; p}\left(t_{1}, t_{2}\right)\right\rangle^{1 / 2}}
$$

(where no summation is intended with respect to subscripts) yields

$$
\begin{gathered}
\frac{d\left\langle x_{i ; p}^{\prime}\left(t_{1}, t_{2}\right) x_{j ; p}^{\prime}\left(t_{1}, t_{2}\right)\right\rangle}{d t_{2}}=\left\langle x_{i ; p}^{\prime}\left(t_{1}, t_{2}=0\right) u_{j ; p}^{\prime}\left(t_{1}, t_{2}\right)\right\rangle+\left\langle x_{j ; p}^{\prime}\left(t_{1}, t_{2}=0\right) u_{i ; p}^{\prime}\left(t_{1}, t_{2}\right)\right\rangle+ \\
\int_{0}^{\tau}\left\{\left\langle u^{\prime 2}{ }_{i ; p}\left(t_{1}, t_{2}\right)\right\rangle^{\frac{1}{2}}\left\langle u^{\prime 2}{ }_{j ; p}\left(t_{1}, t_{2}\right)\right\rangle^{\frac{1}{2}} R_{i j ; p}^{L}\left(\tau^{\prime}, t_{2}\right)+\left\langle u_{j ; p}^{\prime 2}\left(t_{1}, t_{2}\right)\right\rangle^{\frac{1}{2}}\left\langle u_{i ; p}^{\prime 2}\left(t_{1}, t_{2}\right)\right\rangle^{\frac{1}{2}} R_{j i ; p}^{L}\left(\tau^{\prime}, t_{2}\right)\right\} d \tau^{\prime}
\end{gathered}
$$

Equation 8 and 6 are sufficient expressions for the time dependence of the variance in particle position. Neither equation is very useful, however, since the joint velocity correlations are not clearly related to gas properties or known particle properties.

A useful relationship between the correlations in Eq. 6 and 8 and known properties can be developed. Subtracting Eq. 3 from Eq. 2 yields an expression for the residence-time variation in the perturbation velocity as a function of particle and gas properties.

$$
\frac{d u_{i ; p}^{\prime}\left(t_{1}, t_{2}\right)}{d t_{2}}=\beta\left(t_{2}\right)\left(u_{i ; g}^{\prime}\left(t_{1}, t_{2}\right)-u_{i ; p}^{\prime}\left(t_{1}, t_{2}\right)\right)+A_{i}^{\prime}\left(t_{1}, t_{2}\right)
$$

The general solution to this equation can be written as

$$
\begin{aligned}
u_{i ; p}^{\prime}\left(t_{1}, \tau\right)= & \exp \left[-\int_{0}^{\tau} \beta\left(t^{\prime}\right) d t^{\prime}\right] \\
& \left\{u_{i ; p}^{\prime}\left(t_{1}, t_{0}\right)+\int_{0}^{\tau}\left[\beta\left(t^{\prime}\right) u_{i ; g}^{\prime}\left(t_{1}, t^{\prime}\right)+A_{i}^{\prime}\left(t_{1}, t^{\prime}\right)\right] \exp \left[\int_{0}^{t^{\prime}} \beta\left(t^{\prime \prime}\right) d t^{\prime \prime}\right] d t^{\prime}\right\}
\end{aligned}
$$


where $u_{i ; p}^{\prime}\left(t_{1}, t_{0}\right)$ represents the initial particle velocity perturbation $\left(u_{i, p}^{\prime}\left(t_{1}, t_{2}=0\right)\right)$ and $\tau=t_{2}-t_{0}$. Variables of integration are indicated by either single or double primes in Eq. 10. The ensemble average of the product of Eq. 10 and $u_{j ; p}^{\prime}\left(t_{1}, t_{2}\right) /\left[\left\langle u_{i: p}^{\prime 2}\left(t_{1}, t_{2}\right)\right\rangle^{1 / 2}\left\langle u^{\prime 2}{ }_{j ; p}\left(t_{1}, t_{2}\right)\right\rangle^{1 / 2}\right]$ yields

$$
\begin{aligned}
& R_{i j ; p}^{L}\left(\tau, t_{2}\right) \equiv \frac{\left\langle u_{i ; p}^{\prime}\left(t_{1}, \tau\right) u_{j ; p}^{\prime}\left(t_{1}, t_{2}\right)\right\rangle}{\left\langle u_{i ; p}^{\prime 2}\left(t_{1}, t_{2}\right)\right\rangle^{\frac{1}{2}}\left\langle u^{\prime 2}{ }_{j ; p}\left(t_{1}, t_{2}\right)\right\rangle^{\frac{1}{2}}}=\exp \left[-\int_{0}^{\tau} \beta\left(t^{\prime}\right) d t^{\prime}\right]\left\{\frac{\left\langle u_{i ; p}^{\prime}\left(t_{1}, t_{0}\right) u_{j ; p}^{\prime}\left(t_{1}, t_{2}\right)\right\rangle}{\left\langle u_{i ; p}^{\prime 2}\left(t_{1}, t_{2}\right)\right\rangle^{\frac{1}{2}}\left\langle u^{\prime 2}{ }_{j ; p}\left(t_{1}, t_{2}\right)\right\rangle^{\frac{1}{2}}}\right. \\
& \left.+\int_{0}^{\tau}\left[\frac{\beta\left(t^{\prime}\right)\left\langle u_{i: g}^{\prime}\left(t_{1}, t^{\prime}\right) u_{j: p}^{\prime}\left(t_{1}, t_{2}\right)\right\rangle}{\left\langle u^{\prime 2}{ }_{i ; p}\left(t_{1}, t_{2}\right)\right\rangle^{\frac{1}{2}}\left\langle u_{j ; p}^{\prime 2}\left(t_{1}, t_{2}\right)\right\rangle^{\frac{1}{2}}}+\frac{\left\langle A_{i}^{\prime}\left(t_{1}, t^{\prime}\right) u_{j ; p}^{\prime}\left(t_{1}, t_{2}\right)\right\rangle}{\left\langle u_{i: p}^{\prime 2}\left(t_{1}, t_{2}\right)\right\rangle^{\frac{1}{2}}\left\langle u_{j ;: p}^{\prime 2}\left(t_{1}, t_{2}\right)\right\rangle^{\frac{1}{2}}}\right] \exp \left[\int_{0}^{t^{\prime}} \beta\left(t^{\prime \prime}\right) d t^{\prime \prime}\right] d t^{\prime}\right\}
\end{aligned}
$$

This defines the normalized Lagrangian particle correlation function. Some limiting forms of the equation will be considered, concluding with one that is applicable to the type of information available from common, two-equation turbulence models.

\section{Markovian Approximation}

The Lagrangian velocities are stationary with respect to residence time if the flow field is homogeneous and the particles are injected at their equilibrium velocities. One property of stationary variables is that their correlations, such as $R^{L}$, are even functions of the difference in time, $\tau$, and independent of (residence) time itself 15,18 . A residence time of zero is a particularly conventient choice for evaluating $t_{2}$. The rms perturbations are also independent of residence time. If the magnitudes of the correlations with gas velocity and random perturbations decay instantly, a classical Markovian system ${ }^{19}$ obtains in which

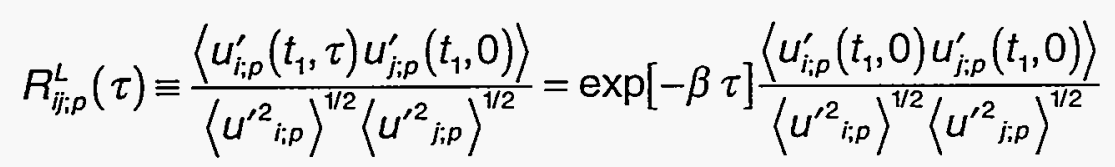

where $\tau$ is used to represent residence time difference. For the principal diagonal of this tensor ( $i=$ J) the second factor on the right becomes unity and Eq. 12 defines the autocorrelation function, 
which is seen to be an exponential decay. This classical result relates to early progress in describing Brownian motion (effect of $A$ is ignored).

\section{Fluid Particle (Gas) Dispersion}

If the particles are assumed to be fluid particles $(\beta \rightarrow \infty)$ dispersing in a homogenous flow field,

$$
R_{i j ; p}^{L}(\tau)=\frac{\left\langle u_{i ; g}^{\prime}\left(t_{1}, \tau\right) u_{j ; g}^{\prime}\left(t_{1}, 0\right)\right\rangle}{\left\langle u^{\prime 2}{ }_{i ; g}\right\rangle^{1 / 2}\left\langle u^{\prime 2}{ }_{j ; g}\right\rangle^{1 / 2}}=R_{i j ; g}^{L}(\tau)
$$

In nonstructured turbulence, the autocorrelation function for the gas can also often be approximated by an exponential decay. These results relate to turbulent dispersion work initiated by Taylor and carried on by others $11,12,20,21$.

\section{Parameter Definition for two-equation turbulence models}

Two-equation descriptions of turbulence such as the $k-\varepsilon$ model predict a turbulent kinetic energy and a decay rate. If the particles are entrained in a gas with velocity fluctuations of frequency $s$ and exponentially changing magnitude with time constant $1 / r$, then the following relationship can be shown between the magnitude of the rms velocity fluctuations of the gas and particles 16

$$
\frac{\left\langle v_{i, p}^{\prime 2}\left(t_{1}, t_{2}\right)\right\rangle}{\left\langle v_{i ; g}^{\prime 2}\left(t_{1}, t_{2}\right)\right\rangle}=\frac{\sigma_{i, v_{p}}^{2}}{\sigma_{i, v_{g}}^{2}}=\frac{\beta^{2}}{(\beta+r)^{2}+s^{2}}
$$

This result assumes that the decay rate, $r$, is slow enough that the particles experience several fluctuations before the turbulence becomes zero. The rate of change of turbulent intensity, $r$, is negative if the turbulence is decaying. The predictions of Eq. 4 in the limits of small and large particles and in the limits of homogeneous and inhomogeneous turbulence are as follows: (1) large particles $(\beta<<)$ in nearly homogeneous flows $(\beta<<|r|)$ are not significantly impacted by gas

phase fluctuations; (2) small particles $(\beta>>s)$ in nearly homogeneous flows $(\beta<<|r|)$ are dominated by gas phase fluctuations; (3) all particles in decaying turbulent flow fields $(r<0)$ 
have the potential of exhibiting gas-phase induced velocity fluctuations greater than those of the gas in the local region.

If the residence time dependence of the gas-phase correlations scales with turbulent intensity and the effects of random forces are ignored, the correlation function is can be simplified for use in typical turbulence models. The influence of the molecular bombardment on the particles is small compared with that of the gas fluctuations and are ignored. These assumptions allow an approximate form of the correlation function to be written as

$$
\frac{d\left\langle x_{i ; p}^{\prime 2}\left(t_{1}, t_{2}\right)\right\rangle}{d t_{2}}=\int_{0}^{t_{2}}\left\{2\left\langle u_{i ; g}^{\prime 2}\left(t_{1}, t\right)\right\rangle \frac{\left.\frac{\beta^{2}}{(\beta+r)^{2}+s^{2}}+\int_{0}^{t} \beta R_{i ; ; g}^{L}\left(t^{\prime}\right) \exp \left(-\int_{0}^{t^{\prime}} \beta\left(t^{\prime \prime}\right) d t^{\prime \prime}\right) d t^{\prime}\right)}{\exp \left(\int_{0}^{t} \beta\left(t^{\prime}\right) d t^{\prime}\right)}\right\} d t
$$

where all particles are assumed to be injected in the flow from a point source at $x=0$ and at residence time zero. No summation of indices is implied. The off-axis components of the covariance tensor are ignored if no information on correlations between different velocity components is available, as is usually the case for simple turbulence models.

The gas correlation function is also typically not available. In such cases, a Markovian approximation is made for the gas as follows

$$
R_{i i ; g}^{L}(t)=\exp \left[-\int_{0}^{t} \tau_{g}\left(t^{\prime}\right) d t^{\prime}\right]
$$

where

$$
\tau_{g}=\frac{C_{\mu}^{3 / 4} k^{3 / 2}}{\varepsilon\left(\frac{2 k}{3}\right)^{1 / 2}}
$$


The mean and variances of the pdf are now fully specified, and the information available from the gas-phase turbulence model is exhausted. Given more information, the off-axis components of the covariance matrix and possibly higher-order moments of the mean could be specified. However, there are theoretical reasons to believe that the pdf eventually becomes a multivariate normal distribution, in which case the mean and covariance matrix are all that is required to fully specify it. The information at hand should be a reasonable approximation to the actual rate of dispersion.

The description of particle dispersion presented above differs from classical analyses that invoke diffusion coefficients. Such a diffusion coefficient, and the associated concentration gradient, are not used in the STP model. Since the complete solution to the dispersion problem is available, the diffusion coefficient could be calculated from the results. Such a calculation results in

$$
\begin{gathered}
D_{i j ; p}^{*}\left(t_{2}\right)= \\
\frac{1}{2} \int_{0}^{t_{2}}\left\{\left\langle u^{\prime 2}{ }_{i ; \rho}\left(t_{1}, t_{2}\right)\right\rangle^{\frac{1}{2}}\left\langle u_{j ; \rho}^{\prime 2}\left(t_{1}, t_{2}\right)\right\rangle^{\frac{1}{2}} R_{i j ; p}^{L}\left(t, t_{2}\right)+\left\langle u_{j ; p}^{\prime 2}\left(t_{1}, t_{2}\right)\right\rangle^{\frac{1}{2}}\left\langle u_{i ; p}^{\prime 2}\left(t_{1}, t_{2}\right)\right\rangle^{\frac{1}{2}} R_{j i ; p}^{L}\left(t, t_{2}\right)\right\} d t
\end{gathered}
$$

This diffusion coefficient is the proportionality constant between the negative of the local particle concentration gradient and the local particle mass flux. However, it has several properties that are not normally associated with diffusion coefficients. For example, it has a clear time dependence in all cases where particles are injected from a point source. It is also potentially negative valued. Finally, in can not be calculated from local conditions in the flow alone. The asterisk is used to distinguish this coefficient from the more classical concept. The reasons for these differences are discussed later.

\section{Comparison of STP Results with Analytical and Experimental Data}

The STP model has been compared with analytical and experimental data. In all cases, the environment in which the particles are dispersing is either well characterized or, in the case of the analytical results, precisely defined. This has the advantage of ensuring that the comparisons reflect dispersion calculations rather than ability to model or measure turbulent environments. It 
has the disadvantage of being limited to very simple flow configurations. The purpose in performing the comparisons is to develop insight into the dispersion process generally.

The solution to the diffusion equation is well established under the conditions of a point source with a constant velocity in one direction and a constant (and known) diffusion coefficient. This solution has been used by others to evaluate particle dispersion models. Results from two such models (SSF 1 and SSF 2) are shown in Figure 5 together with the analytical results (shown as points) and the prediction of the STP model (indicated by the authors' names) 9,10 . Specification of the distance and time between random velocity perturbations is the principal difference between the two SSF models. The two stochastic separated flow (SSF) models indicated are similar in some respects to the STP model except that the statistical averaging is done by launching particles into the flow stream and integrating their trajectories in a Monte Carlo fashion. Results from many trajectories are averaged. By comparison, the STP model averages the equations to produce a statistical description of the cloud of particles. The advantage to the SSF approach is that nonlinearities in the equations can be dealt with more accurately than in the STP model. The advantages to the STP approach are computational efficiency and formalized relationships between gas and particle turbulence properties. The principal weakness of both models is a lack of detailed gas turbulence data (Lagrangian velocity covariance tensors, for example).

In this idealized case, where diffusion coefficients are constant and known, the STP model predicts the analytical result. The value of the integrand in Eq. 18 eventually becomes zero, and integral is a constant. This constant is the diffusion coefficient. If the integrand is adjusted such that it rapidly decreases to zero and its integral has the proper magnitude, the STP model produces the same result as the analytical solution. The result is obtained in a single simulation of a particle cloud. Monte Carlo techniques, by their very nature, require several thousand trajectory calculations to produce a well-behaved solution. A cloud simulation requires about three times as much computational effort as a single Monte Carlo trajectory calculation. 
The value of the mean particle position is easily computed in a homogeneous, uniform flow. To a large extent, turbulent particle dispersion is predicted by time development of the covariance matrix. A second set of data that characterize this time development are derived from dispersion of liquid in liquid (no slip velocity) ${ }^{22}$. These data are illustrated in Figure 6 . The data represent the cross-stream variance in particle position as a function of normalized time for a large number of different flow conditions. The normalization constant is the integral time scale, which is the area under the $R_{i j}$ curve between the point of release and the time the curve becomes identically zero. If a Markovian autocorrelation function is used, parameterized by this (measured) time constant, and if the flow is homogeneous, an analytical solution to the time development of the variance can be derived. This function is given by

$$
x_{i i}^{\prime 2}=2 v_{i i}^{\prime 2}\left\{\frac{t_{2}}{\tau}-\frac{1}{\tau^{2}}\left[1-\exp \left(-\tau t_{2}\right)\right]\right\}
$$

where $\tau$ represents the integral time scale. This function is illustrated, together with the data in Figure 6. Both data and function were normalized by the rms velocity fluctuations and measured integral scales were used to normalize the data.

A close examination of Eq. 18 indicates that the diffusion coefficient is proportional to the derivative of the variance, or more properly, the tensor components are proportional to the derivative of the covariance tensor. The data in Figure 6 show that this diffusion coefficient is clearly time dependent up to a normalized time of about 2.5 , even though the flow is essentially homogeneous. A turbulence model that bases its calculations on the values of local variables alone could not capture this effect. The agreement between the data and the STP model results from (1) modeling the process in a physically meaningful way, and (2) having access to accurate turbulence properties.

The time dependence of the dispersion process and diffusion coefficient are closely related to the time dependence of $R_{i j}$. In the absence of better information, the STP model uses the Markovian approximation for $R_{i j}$. Classical data under well-characterized conditions are available to evaluate 
the adequacy of this assumption. Figure 7 illustrates the measured values of autocorrelations for particles with a range of relaxation times $(1.7$ to $49 \mathrm{msec})$ dispersing in a well-characterized field of

grid turbulence 23 . The data are all normalized with the respective measured integral scales of the particles. Also indicated in the figure is the Markovian autocorrelation function. There are minor differences between the Markovian autocorrelation function and the data. In particular, the data indicate a slightly negative value between the normalized times of 4 and 6 . The Markovian exponential decay is nonnegative and monotonically decreasing. Aside from these minor differences, the agreement is good.

\section{Implications for Particle Dispersion Generally}

Most flow environments of practical concern contain shear layers, recirculation zones, and other features that make the dispersion problem much more complicated than in those discussed in the previous section. If the details of the gas turbulence field are known, the STP model offers a reasonably rigorous approach to predicting the particle dispersion. In most cases, the gas flow is not well characterized. However, the results from the comparisons with the simple flows previously discussed allow some comment to be made on the general nature of particle dispersion in the great majority of practical flows.

Classical Fickian diffusion asserts that a diffusion flux is proportional to a concentration gradient and a diffusion coefficient, the latter being independent of residence time in a steady flow. The data and theory previously presented indicate that turbulent particle dispersion cannot be described by such an approach until the correlation tensor decays to a steady value of zero. The integral time scale is an indication of the time required before a group of initially concentrated particles is no longer influenced by their initial conditions and the correlation tensor decays to zero. In combustion flows with which the authors are most familiar, this integral time scale is on the order of one second. This is long compared to other time scales of interest in such flows. Therefore, most of the relevant dispersion occurs before the diffusion coefficient becomes independent of time. 
There are several indications of more complex dispersion. One of the most vivid is countergradient diffusion. The physical origins of counter-gradient diffusion are difficult to appreciate when viewed from a reference frame of Fickian diffusion. If viewed from the statistics of turbulent fluctuations, Equation 11 shows how negative gas-phase velocity correlations can cause the particle correlation function to become negative. If the magnitude and length of the negative correlation is sufficient to cause the integral in Eq. 18 to become negative, counter-gradient diffusion will occur. An illustration of such behavior is included as Figure 8.

The residence-time dependence of the correlation function complicates the description of particle dispersion from an Eulerian reference frame. Figure 9 illustrates an example where particles are injected at two points separated in time by less than the integral scale of the turbulence. Under such conditions, identical particles at identical locations in identical environments will exhibit different diffusion coefficients because of this residence-time dependence. Therefore, particle dispersion under such conditions cannot be described as a function of local properties only.

\section{Conclusions}

An approach to describing particle transport in turbulent flows has been demonstrated based on an extension of stochastic process modeling and turbulence theory. The model requires no adjustable parameters beyond specification of the turbulent flowfield itself and is independent of any particular turbulence model. Approximations for the parameters required in the model are presented in terms of parameters from the $k-\varepsilon$ gas-phase turbulence model and particle properties.

This stochastic transport of particles (STP) model does not depend on specification of diffusion coefficients or concentration gradients. Indeed, the model indicates that diffusion coefficients in turbulent flows typically are time dependent for times long relative to many important process time constants. This time dependence is shown to be exhibited in a wide variety of flows. For simple, non-structured flows, a Markovian approximation is shown to represent the correlation function 
adequately. For more complex flows, the STP model can take advantage of more detailed gasphase turbulence parameters and predict, for example, counter-gradient diffusion. Implications of the results for turbulent dispersion generally are also discussed.

\section{Acknowledgments}

Significant portions of this work were completed when both authors were associated with Brigham Young University, Provo, UT. Some additional work was performed at Sandia National Laboratories in conjunction with coal-water slurry modeling. The work at both institutions was supported by the U.S. Department of Energy through the Morgantown Energy Technology Center.

\section{References}

1. Faeth, G.M. Progress in Energy and Combustion Science 9, 1- (1983).

2. Kuo, K.K. Principles of Combustion (Wiley, New York, 1986).

3. Hinze, J.O. Turbulence (McGraw-Hill, New York, 1975).

4. Goldschmidt, V.W., Householder, M.K., Ahmadi, G. and Chuang, S.C. Progress in Heat and Mass Transfer 6, 487 (1972).

5. Lumely, J.L. Topics in Applied Mechanics (eds. Bradshaw, P.) (Springer-Verlag, Berlin, 1978).

6. Chigier, N.A. Progress in Energy and Combustion Science 2, 97 (1976).

7. Lilly, G.P. Industrial and Engineering Chemistry, Fundamentals 12, 268 (1973).

8. Crowe, C.T. Transactions of ASME, Journal of Fluids Engineering 104, 197 (1982).

9. Shuen, J.-S., Chen, L.-D. and Faeth, G.M. AIChE Journal 29, 167 (1983). 
10. Gosman, A.D. and Ioannides, E. Aspects of Computer Simulations of Liquid-Fueled Combustion (AIAA, 1981).

11. Taylor, G.I. Proceedings of the London Mathematical Society 20, 196-211 (1921).

12. Batchelor, G.K. Australian Journal of Scientific Research A2, 437 (1949).

13. Csanady, G.T. Turbulent Diffusion in the Environment (D. Reidel Publishing Company, Dordrecht, Holland, 1973).

14. Monin, A.S. and Yaglom, A.M. Statistical Fluid Mechanics (MIT Press, Cambridge, 1971).

15. Gardiner, C.W. Handbook of Stochastic Methods for Physics, Chemistry, and the Natural Sciences (Springer-Verlag, Berlin, 1985).

16. Baxter, L.L. Turbulent Transport of Particles (Brigham Young University, 1989).

17. Abbot, M.F., Moza, A.K. and Austin, L.G. Fuel 60, 1065-1072 (1981).

18. Papoulis, A. Probability, Random Variables, and Stochastic Processes (McGraw-Hill, New York, 1984).

19. Ethier, S.N. and Kurtz, T.G. Markov Processes (Wiley, New York, 1986).

20. Taylor, G.I. Proccedings of the Royal Society of London 135, 685-706 (1932).

21. Batchelor, G.K. Journal of Fluid Mechanics 3, 67 (1957).

22. Kalinske, A.A. and Pien, C.L. Industrial and Engineering Chemistry 36, 220 (1944).

23. Snyder, W.H. and Lumley, J.L. Journal of Fluid Mechanics 48, 41-71 (1971). 
Figure 1. Illustration of the development of a Lagrangian pdf for particle position in an arbitrary coordinate system. At short residence times the mean particle location is near the point of injection (the origin in this case) as represented by the peak in the particle position pdf. The pdf is confined to a relatively small region of the flow domain. Flow is directed primarily in the $+x$ direction. Compare with Figures 2 and 3.

Figure 2. Illustration of the development of a Lagrangian pdf in an arbitrary coordinate system. At intermediate residence times the mean particle location is in a central region in the flow domain and the particle position pdf has nonzero values in a significant region of the flow domain. Compare with Figures 1 and 3.

Figure 3. Illustration of the development of a Lagrangian pdf in an arbitrary coordinate system. At long residence times the mean particle location approaches the exit of the flow domain (flow is directed primarily in the $+\mathrm{x}$ direction) and the particle position pdf has nonzero values over most of the flow domain. Compare with Figures 1 and 2.

Figure 4. A Lagrangian view of dispersion in a one dimensional flow. Each circle is a measure of the spatial extent of a pdf for particle position at successive times during the flow. The position of a typical Eulerian computational cell is also illustrated. Particles of many different residence times contribute to the overall population of particles in the cell.

Figure 5. Comparison among several particle dispersion models and an analytical solution.

Figure 6. Comparison of fluid particle dispersion exhibiting Markovian-type dispersion. The diffusion coefficient is proportional to the slope of the data and clearly time dependent. 
Figure 7. Comparison of particle autocorrelation functions, normalized by measured integral scales, and the Markovian autocorrelation. The diffusion coefficient is residence-time-dependent until the autocorrelation function becomes identically zero.

Figure 8. Illustration of the behavior of a diffusion coefficient based on a Markovian process and the diffusion coefficient from more complicated flows, in this case exhibiting counter-gradient diffusion.

Figure 9. Illustration of the significance of a time dependent diffusion coefficient in making Eulerian calculations of particle dispersion. Identical particles in identical environments exhibit different diffusion coefficients at the same location because of the residence-time dependence of the correlations functions.

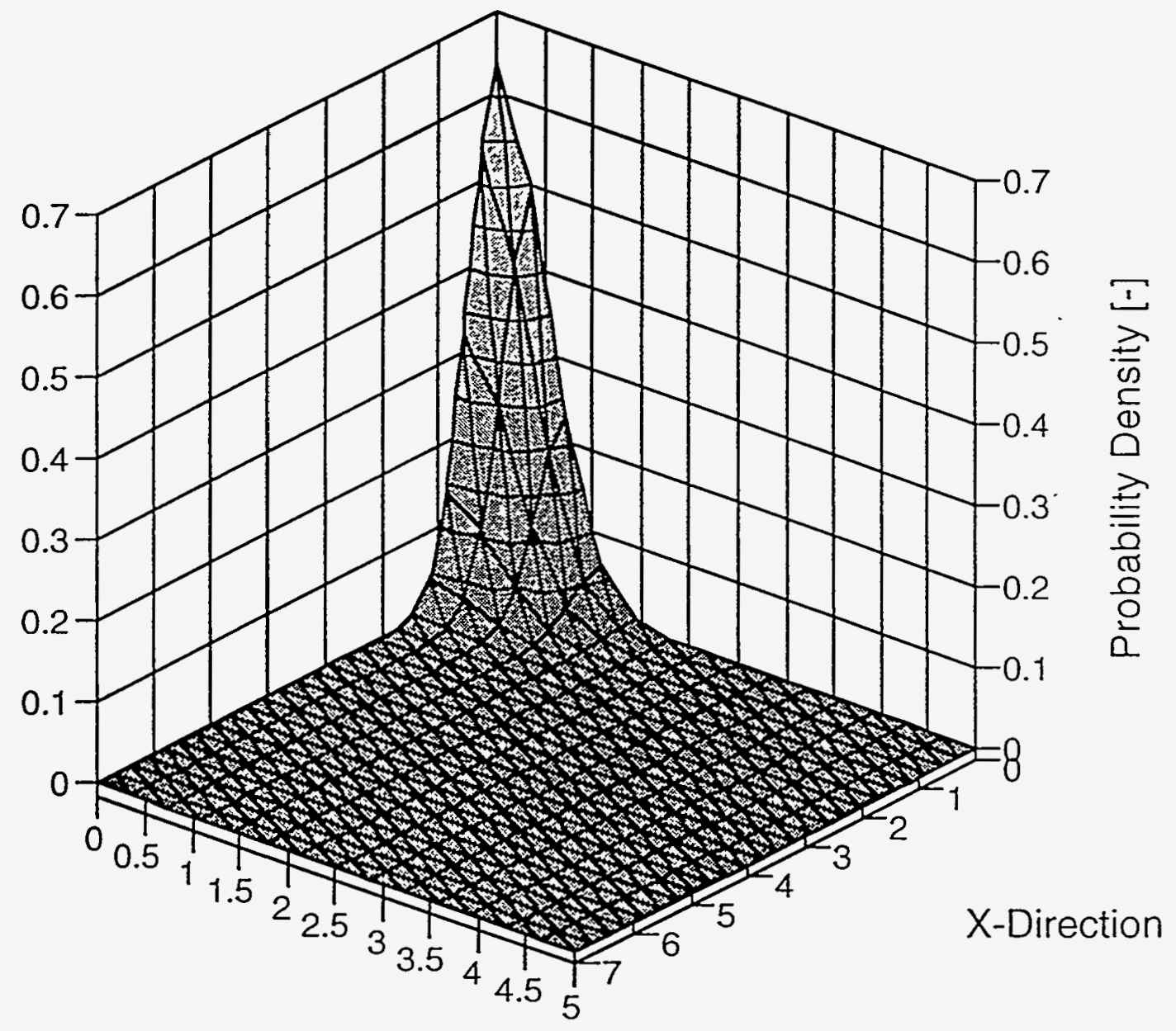

Y-Direction 


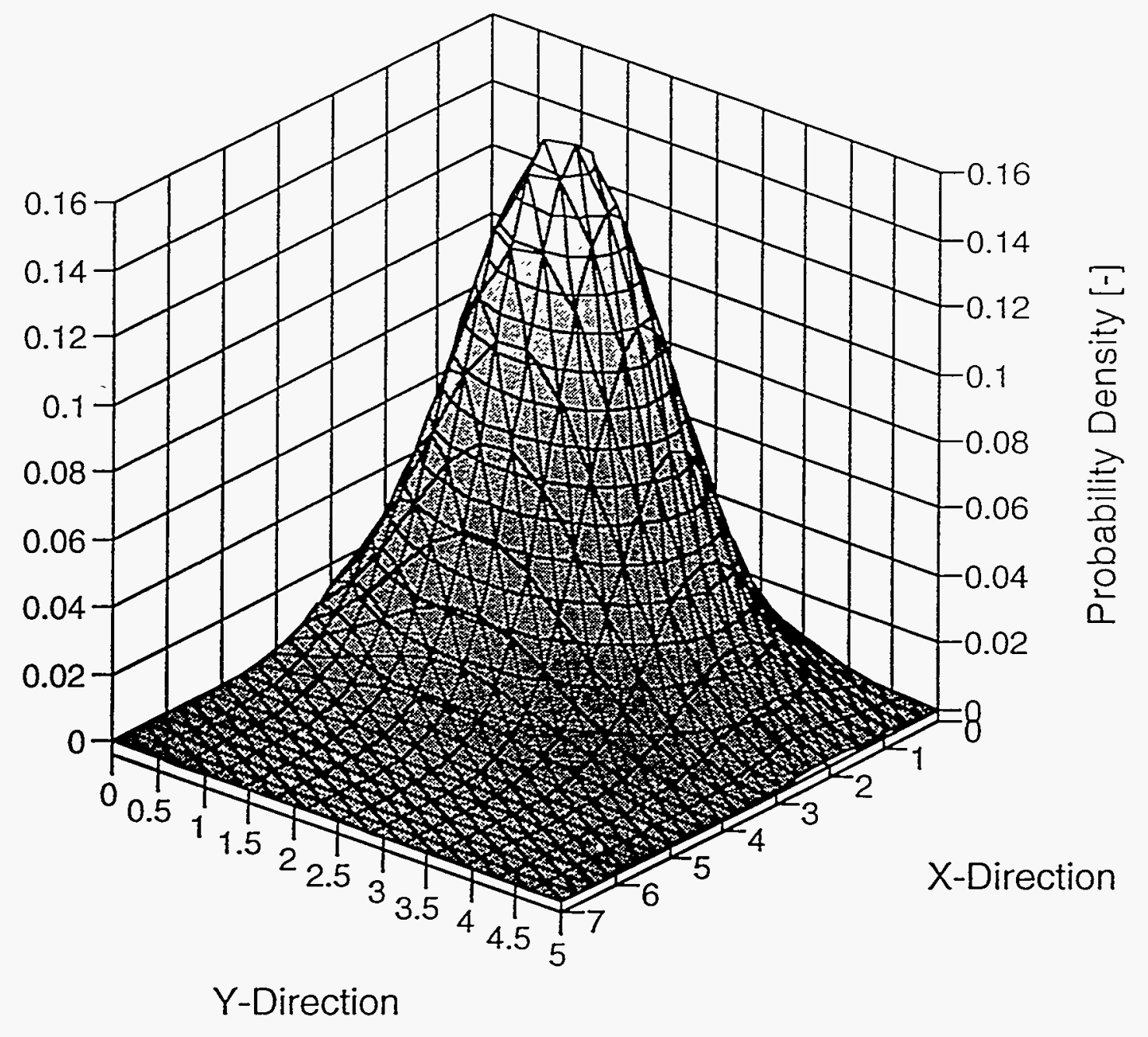




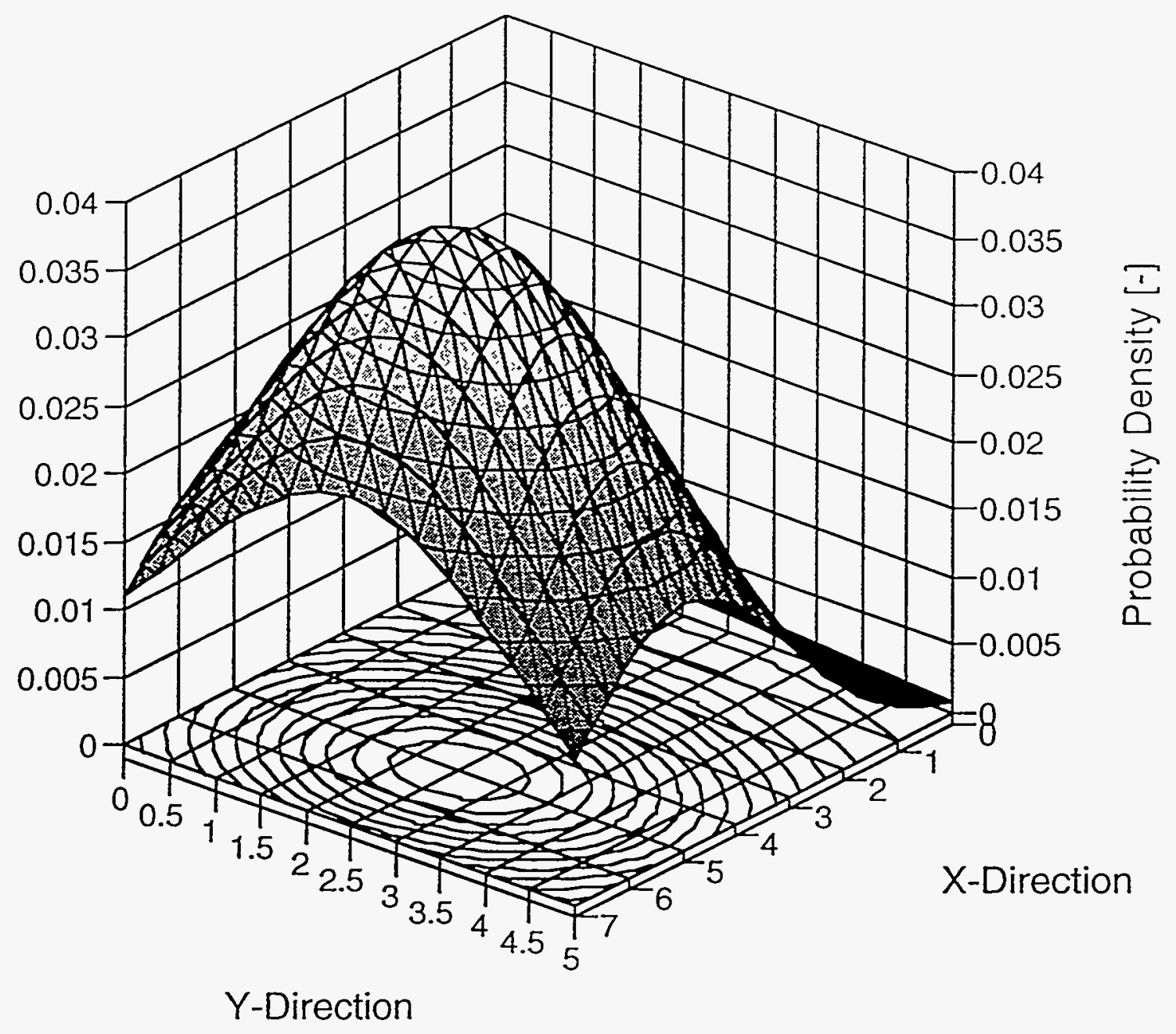




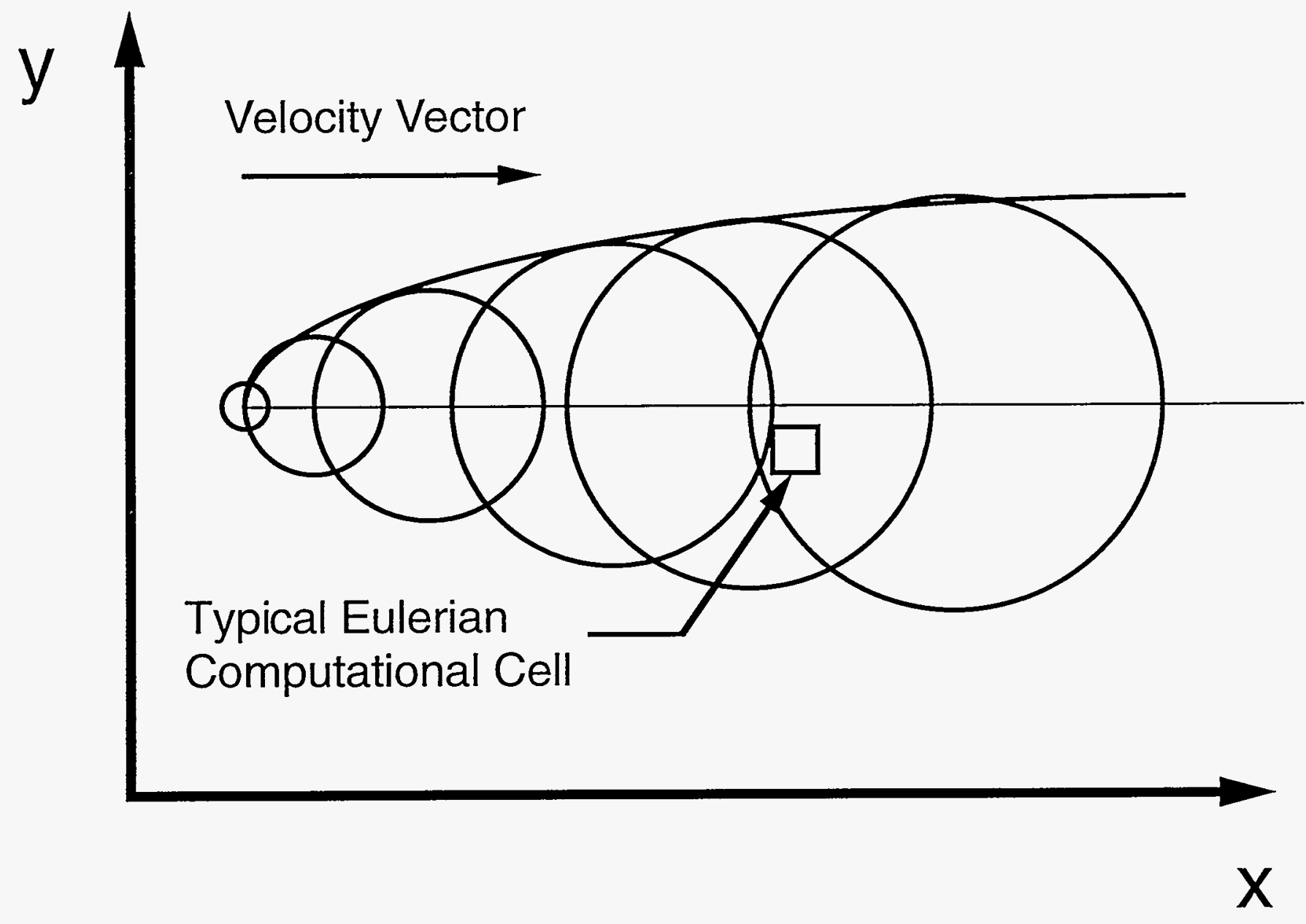




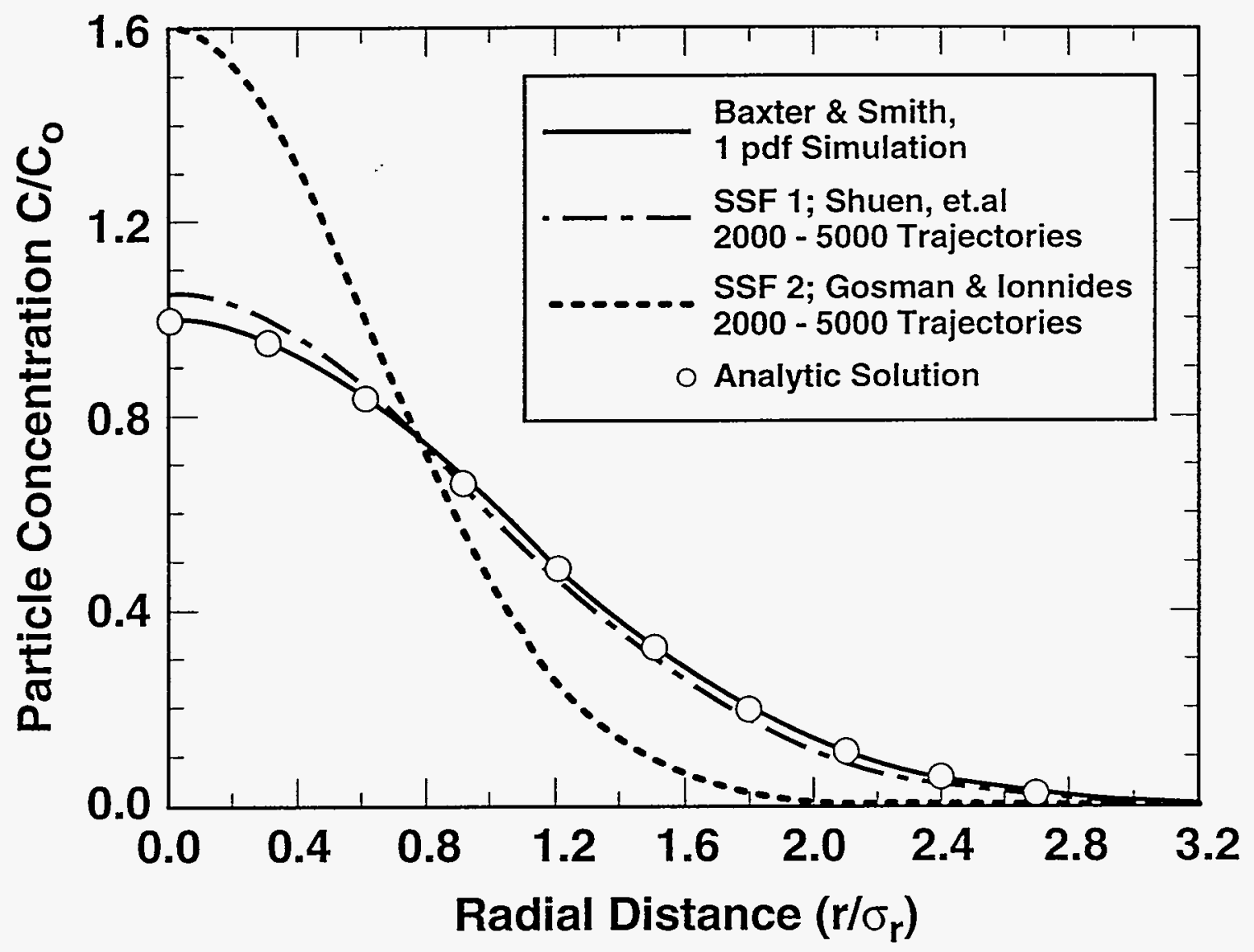




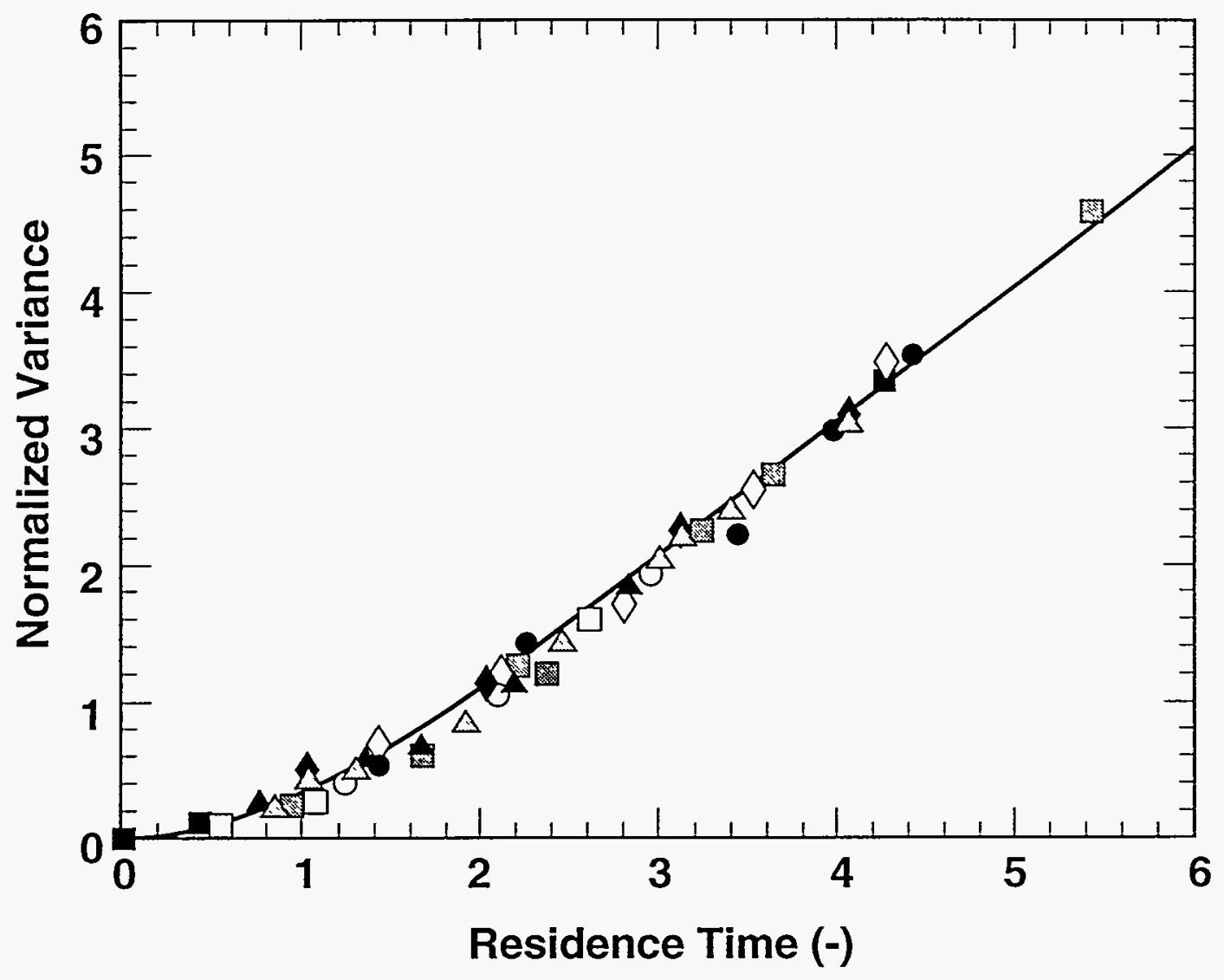




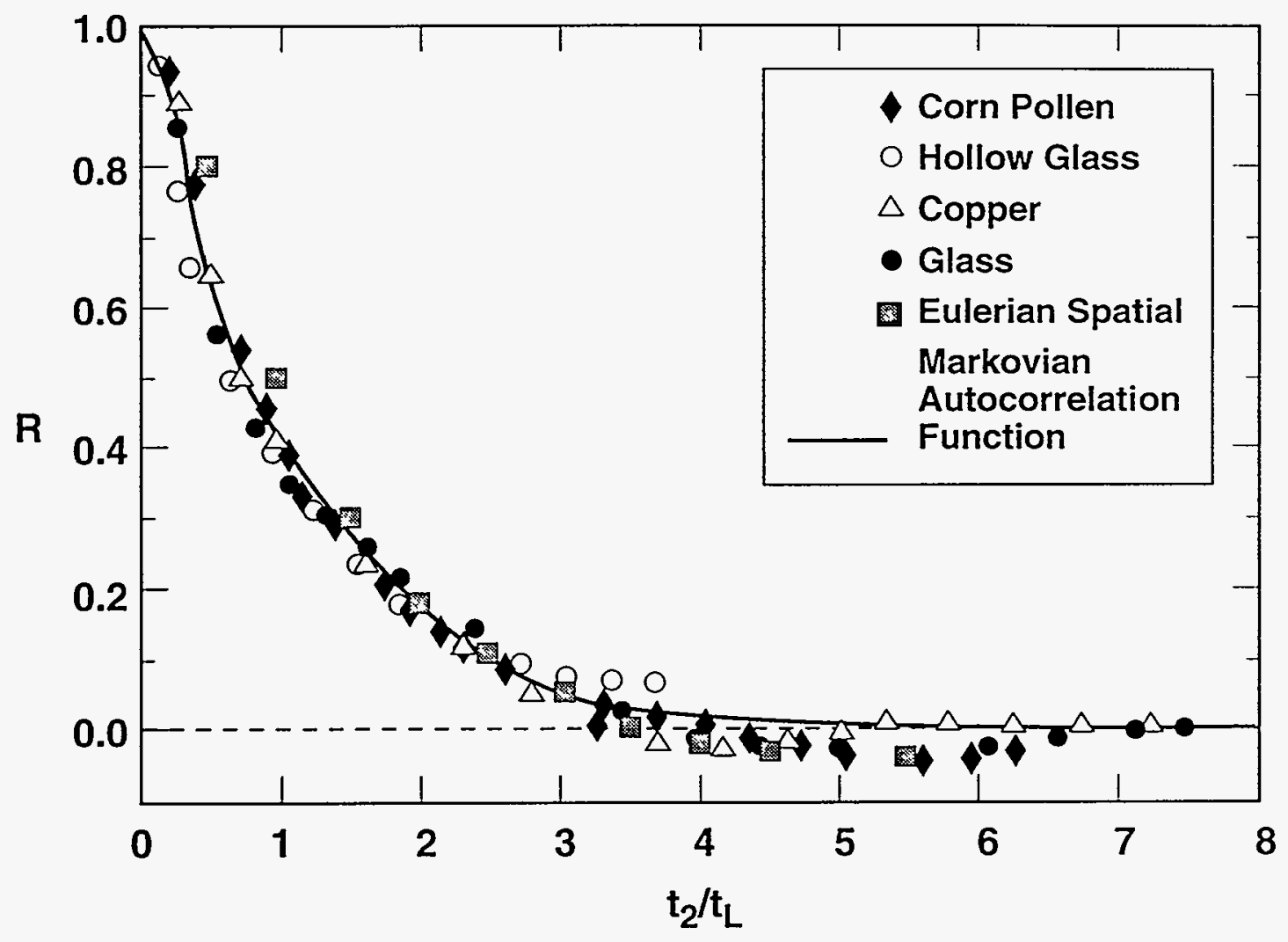




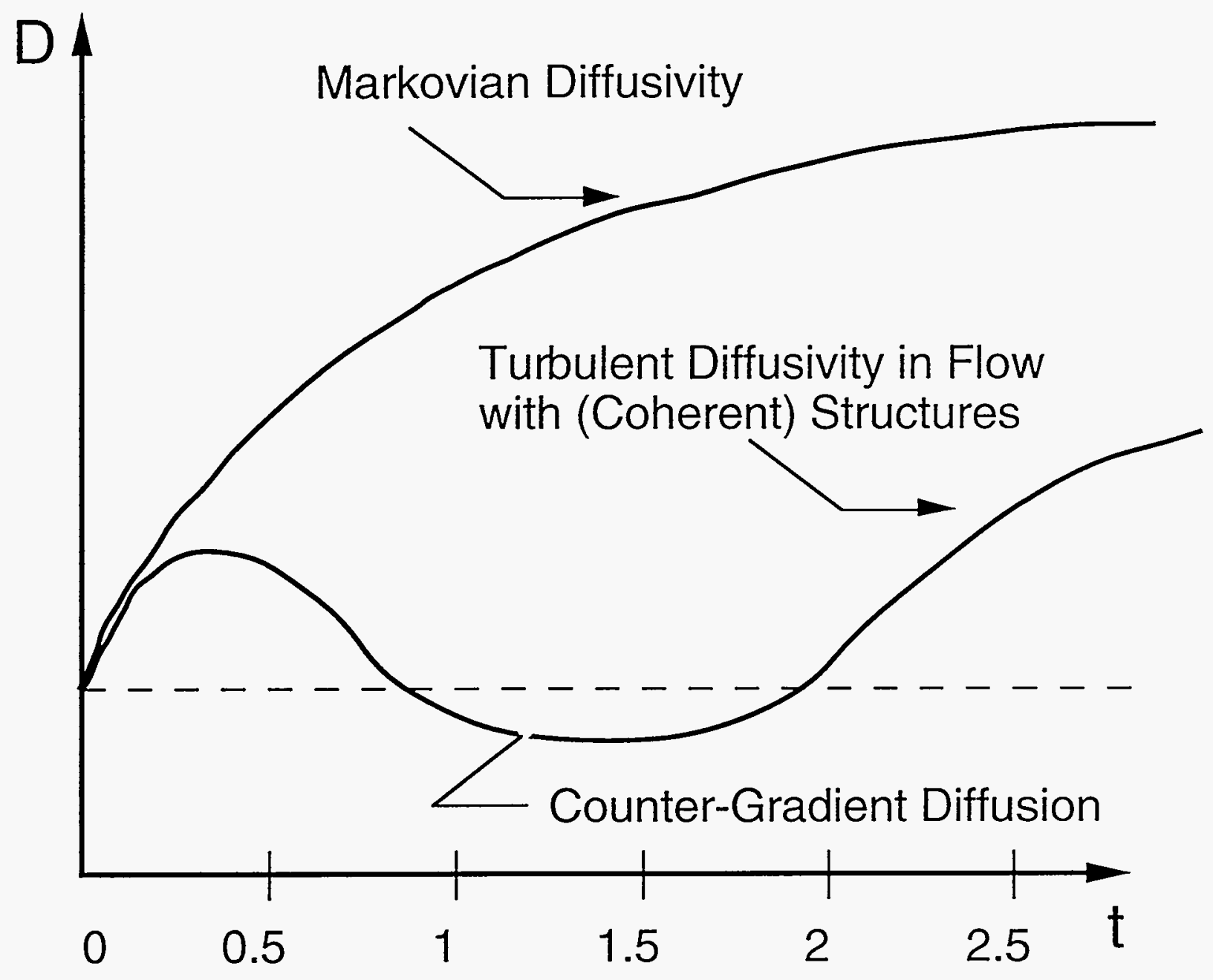


Fixed (Eulerian) Location

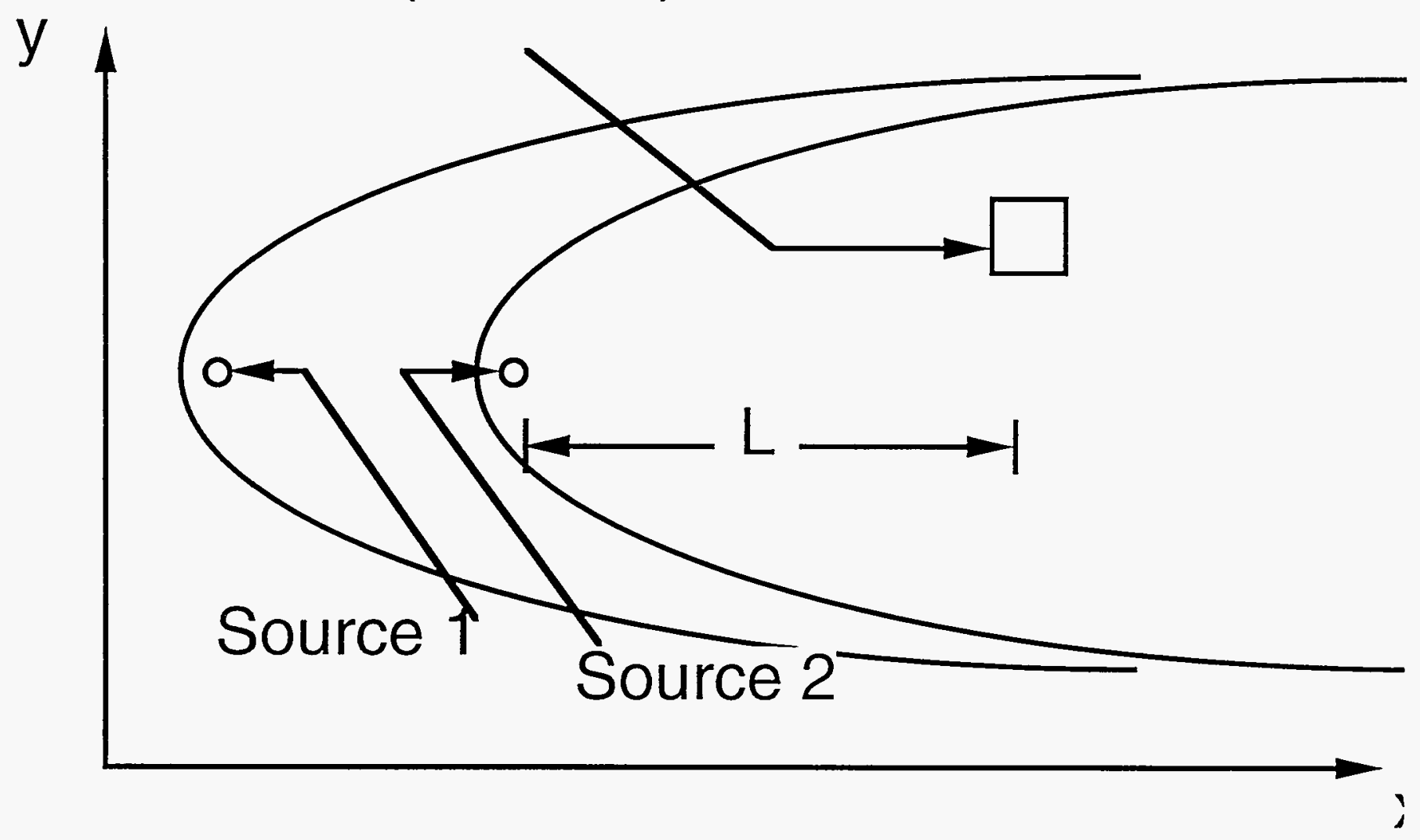

